\title{
Tre progetti per conoscere, approfondire, riflettere
}

L'articolo analizza sinteticamente tre progetti inerenti ai temi dell'antisemitismo e della Shoah, con riferimento a specifiche biografie e vicende di salvataggio avvenute in Emilia Romagna. I progetti, coerenti con le finalità indicate dalla legge istitutiva del Giorno della Memoria e con i principi indicati dalla legge Introduzione dell'insegnamento scolastico dell'educazione civica, offrono importanti risorse per attività didattiche e per la formazione continua degli adulti.

The article briefly analyzes three projects related to the themes of anti-Semitism and the Shoah, with reference to specific protagonists and cases of rescue in Emilia Romagna. The projects, in line with the purposes indicated by the law instituting the Holocaust Memorial Day and with the principles indicated by the law Introducing of the school teaching of civic education, offer important resources for teaching activities and for the continuing education of adults.

\section{Una surreale normalità. Modena e I'Italia al tempo delle leggi razziali}

Una surreale normalità. Modena e l'Italia al tempo delle leggi razziali, web documentario dell'Istituto storico di Modena realizzato nel 2018 da Federico Baracchi e Nico Guidetti, si presenta come un progetto di ricerca molto articolato e completo. La "surreale normalità" a cui il titolo fa riferimento è quella determinata dalle leggi razziali, che costringono la parte di religione ebraica della popolazione italiana ad affrontare una vita quotidiana trasformata in incubo, da 
cui ragione e logica sono state escluse. Gli ambiti di ricerca si sviluppano in una dimensione locale che esemplifica gli avvenimenti italiani, a cui è strettamente collegata. Il web documentario è costruito attraverso una ricca e interessante selezione di materiali audiovisivi d'archivio, in particolare appartenenti all'Istituto Luce, che dialogano con le testimonianze raccolte, con gli approfondimenti dei numerosi esperti coinvolti, con i documenti e le fotografie. Il web documentario è suddiviso in dieci capitoli - Una surreale normalità; Il fascismo degli anni '30; Le caratteristiche dell'ebraismo italiano; L'impatto delle leggi antiebraiche; Angelo Fortunato Formiggini, un editore italiano; Il caso Sinigaglia; La persecuzione nella Repubblica sociale italiana; Gli ebrei modenesi tra il 1943 e il 1945; La fine della guerra e la difficilissima ripresa; L'abrogazione delle leggi antiebraiche - che ricostruiscono ed esaminano il periodo 1938-1945, dalla prima legge razziale del Regime che escludeva gli studenti ed i docenti ebrei dalle scuole fino all'abrogazione della legislazione antisemita.

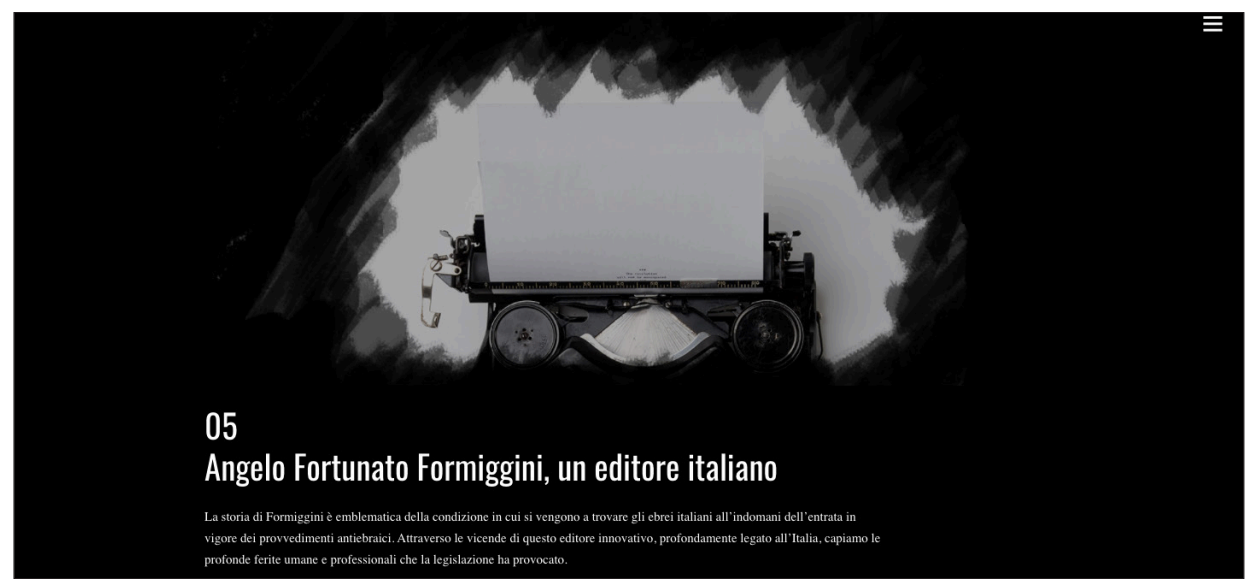

https://unasurrealenormalita.it/capitoli/capitolo-5/

Angelo Fortuna Formiggini, un editore italiano

Scorrendo i titoli dei capitoli, è possibile avere una prima idea dell'ampia ricerca svolta, che comprende anche un focus sull'ebraismo italiano e lo studio di alcune biografie (tra cui è da sottolineare una figura emblematica come quella di Angelo Fortunato Formiggini). Ogni capitolo è completato da una sezione di allegati che consentono ulteriori approfondimenti: sono proposte copie digitali di documenti ufficiali e personali, provvedimenti legislativi, fotografie, copie digitali di riviste e altro materiale. Il web documentario può diventare uno strumento prezioso per la didattica a distanza poiché le diverse sezioni e i tanti materiali presentati per ogni tema possono essere riorganizzati e proposti con una successione risponden- 
te a vari percorsi di studio. Una surreale normalità. Modena e l'Italia al tempo delle leggi razziali può rivelarsi inoltre un'efficace opportunità di formazione per la cittadinanza, in un'ottica di long life learning e di potenziamento dei programmi di elearning rivolti alla popolazione adulta'.

\section{Pietre d'inciampo}

Con il progetto Pietre d'inciampo l'Istituto storico della Resistenza e dell'età contemporanea di Parma concretizza un concetto semplice ma fondamentale, espresso anche da Paul Ricoeur: la storia si svolge non solo nel tempo ma anche nello spazio [Ricoeur 2003]. Documentato nel portale Pietre d'inciampo Parma [https://pietredinciampoparma.it], il progetto si inserisce nell'ambito di un museo diffuso della memoria presente in 17 Paesi europei e realizzato dall'artista tedesco Gunter Demning, con la prima istallazione a Colonia nel 1992, per ricordare le vittime delle persecuzioni naziste. Per preservare dall'oblio le esistenze delle singole persone, ogni piccolo blocco quadrato di pietra, ricoperto di lucido ottone, reca incisi essenziali dati identificativi: data e luogo di nascita, data e luogo di deportazione, data di morte. Posizionata nel marciapiede o sulla strada davanti all'abitazione della persona deportata', la pietra d'inciampo vuole riportare metaforicamente alla propria casa chi è stato travolto dalla violenza delle persecuzio$\mathrm{ni}^{3}$, sottolineando all'interno della dimensione collettiva della Shoah ogni vissuto individuale e contemporaneamente rafforzando i legami esistenti tra le vittime, arrestate e deportate a causa di una stessa appartenenza (ebrei, antifascisti, sinti, omosessuali ...). Riprendendo una riflessione di Patrizia Violi, che analizza i luoghi della memoria soprattutto da un punto di vista semiotico, le pietre d'inciampo «rappresentano in primo luogo l'iscrizione di un valore nello spazio e la sua conservazione e trasmissione nel tempo» [Violi 2014, 84-85]. Occorre provare a definire quali siano i valori affermati da questa particolare espressione di memoria. Oltre a ricordare e commemorare eventi storici, a fare dell'antifascismo e della tutela dei diritti di ogni donna e uomo elementi della struttura urbana, le pietre d'inciampo poste su percorsi quotidiani hanno anche lo scopo di interpellare co-

In base a quanto sollecitato dall'Unione Europea e da altri organismi internazionali.

Più raramente, la pietra d'inciampo viene posta davanti al luogo di lavoro della persona deportata.

«Non è solo un ricordo - ha dichiarato, commossa, Alberta Levi Tamin - è un modo per restituire loro il nome, affermare che non sono dimenticati, riportarli a casa». Cfr. Articolo di Paolo Fallai, "Corriere della sera", 16 ottobre 2011, edizione Roma, p. 16. 
loro che transitano, richiamando l'attenzione e la responsabilità sia sul passato - quasi udendo le parole di Primo Levi: «Voi che vivete sicuri/nelle vostre tiepide case,/voi che trovate tornando a sera/il cibo caldo e visi amici [...]. Meditate che questo è stato: vi comando queste parole./ Scolpitele nel vostro cuore/stando in casa andando per via» - sia sul presente, che non può essere attraversato con indifferenza.

Le pietre d'inciampo assumono dunque un forte significato politico, cioè che attiene alla polis, all'insieme dei cittadini, e l'Istituto storico della Resistenza e dell'età contemporanea di Parma ha coinvolto la città in un progetto che recupera la memoria delle deportazioni di antifascisti, ebrei, internati militari dal capoluogo e dalla provincia. Il portale spiega nell'homepage il significato e le modalità di attuazione del progetto, presentando in una galleria fotografica le attività del periodo 2017-2020. Il portale si può ritenere la versione digitale di uno spazio museale ${ }^{4}$, poiché unisce divulgazione e ricerca scientifica. Dall'homepage la navigazione può proseguire in tre sezioni, ulteriormente suddivise. Una sezione è dedicata alle "Storie" e propone, suddivise in tre gruppi, le biografie di internati militari, ebrei e antifascisti, protagonisti di azioni di salvataggio; ogni biografia è ricostruita con rigore storico ed è corredata da materiale documentale e iconografico di grande interesse; sono indicate le fonti e una bibliografia di riferimento. Le altre sezioni sono rispettivamente dedicate ai "Luoghi" della deportazione - per ognuno dei quali sono fornite informazioni corredate da fotografie - e alla "Didattica"; quest'ultima raccoglie, come è indicato, «i lavori, le produzioni creative e le riflessioni di studentesse e studenti sul tema della deportazione» realizzati attraverso diverse forme espressive: cinema e video, narrazioni, linguaggi pittorici e linguaggi fotografici.

\section{I Giusti. Storie di salvati e di salvatori in Emilia Romagna}

Il film documentario I Giusti. Storie di salvati e di salvatori in Emilia Romagna, promosso dall'Istituto per la Storia della Resistenza e della Società contemporanea in provincia di Reggio Emilia, e realizzato di Andrea Mainardi e Matthias Durchfeld, ha inizio con un lungo piano sequenza che mostra una ferrovia inquadrata dal locomotore di testa di un treno in corsa. Lo spettatore si trova immediatamente tra i passeggeri e osserva l'incedere veloce sui binari. Le immagini della 
ferrovia, dei binari, del treno evocano i vagoni utilizzati per le deportazioni e simboleggiano la modernitàs con cui sono stati pianificati ed attuati il genocidio degli ebrei e lo sterminio di oppositori politici, rom, omosessuali. Il viaggio proposto da Istoreco non ha come destinazione i campi di concentramento ma la salvezza, è un «andare contro una legge ingiusta», come ricordano le parole di don Arrigo Becca ripresentate in apertura ${ }^{6}$.

Il film documentario narra, attraverso le voci di testimoni diretti, le azioni di alcuni uomini e donne emiliano-romagnoli che hanno determinato la salvezza di ebrei perseguitati durante la Shoah e che per questo sono stati nominati "Giusti tra le Nazioni” dal Museo-memoriale di Yad Vashem, fondato nel 1953 per «onorare i sei milioni di ebrei uccisi dai nazisti e dai loro collaboratori [..] e onorare l'eroismo e il coraggio degli ebrei e dei giusti tra le nazioni che hanno rischiato la loro vita per aiutare gli ebrei»?. Cesare Moisè Finzi, raccontando la propria esperienza di studente espulso dalle scuole del Regno d'Italia nel settembre 1938, con poche essenziali parole ricostruisce la persecuzione dei diritti e la persecuzione della vita attuata dal fascismo 8 : «Ecco questo è stato».

Accanto alla narrazione delle vicende delle famiglie Finzi e Rimini, il film documentario ricostruisce quelle della famiglia di Bruno Modena e dei ragazzi ospitati a Villa Emma [www.fondazionevillaemma.org]. Studiare le storie delle italiane e degli italiani nominati "Giusti tra le Nazioni”" consente di approfondire aspetti ancora in parte non adeguatamente esplorati della Shoah in Italia, compreso per esempio il ruolo fondamentale del mondo contadino', che nel film documentario trova espressione nelle vicende degli abitanti del paese di Nonantola che accolgono e nascondono le ragazze e i ragazzi inizialmente ospitati a Villa Emma.

Cfr. Anna Foà, Le diverse tappe della memoria, editoriale, "Gariwo. La foresta dei Giusti", 25 novembre 2020 [https:// it.gariwo.net/editoriali/le-diverse-tappe-della-memoria-22827.html]

Su la storia di don Arrigo Beccari, si veda: Voigt 2002; la pagina dedicata a don Arrigo Beccarri nel sito D'avanti a Villa Emma [http://davantiavillaemma.org/testimonianze/don-arrigo-beccari/]. Altre storie dei "giusti" in Emilia-Romagna sono pubblicate nel sito Giusti Emilia-Romagna. Percorso della memoria tra i "Giusti tra le Nazioni" in Emilia-Romagna [http:// giustiemiliaromagna.it].

Il termine "Giusto" deriva dal Talmud: gentile, giusto è un non ebreo che ha rapporti amichevoli con ebrei. Nel 1963 viene istituita la Commissione dei Giusti per scegliere le persone a cui assegnare l'onorificenza e dedicare un albero in memoria. II primo presidente della commissione è stato Moshe Landau, presidente della Corte che ha condannato a morte Adolf Eichmann nel 1961. Nel 1970 gli è subentrato Moshe Bejski, che ha tenuto la presidenza fino al 1995, segnando il lavoro della commissione con un'interpretazione aperta e articolata della legge del 1953. Attualmente, per mancanza di spazio, gli alberi sono stati sostituiti da iscrizioni su muri appositamente eretti nel giardino.

8 Cfr. Sarfatti 2005.

9 Cfr. Picciotto 2017. 
La legge istitutiva del Giorno della Memoria ${ }^{10}$ pone tra le finalità della commemorazione anche il ricordo di «coloro che, anche se in campi e schieramenti di-

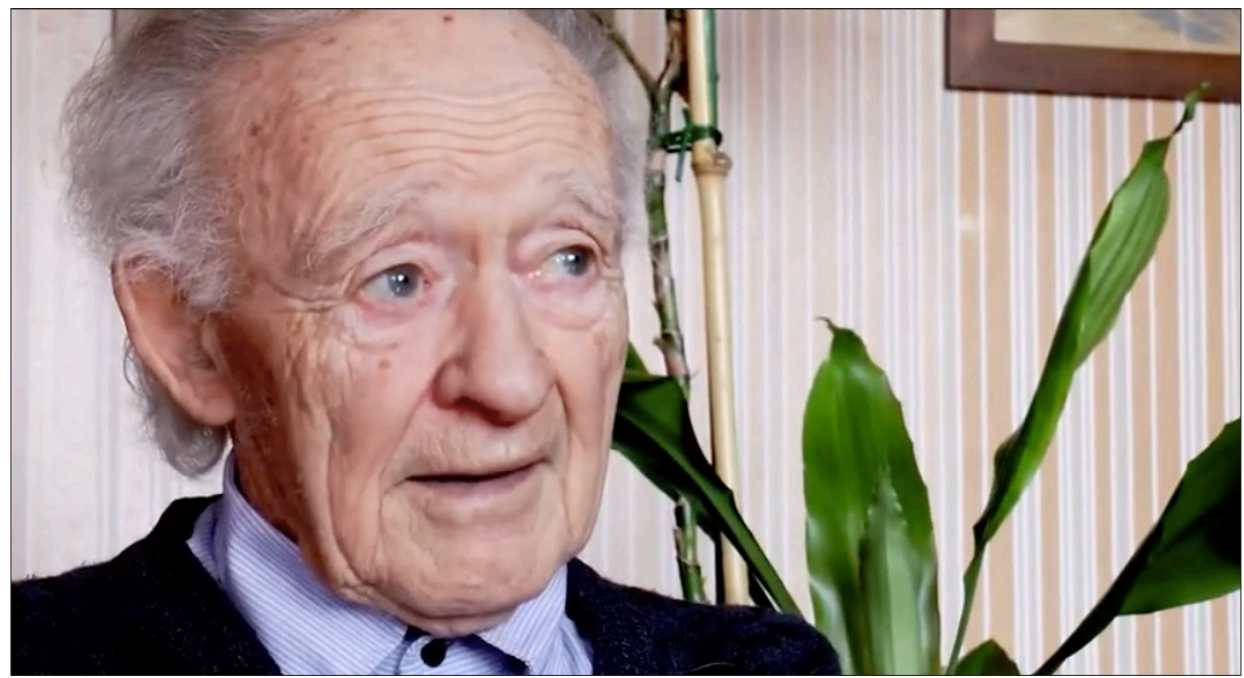

https://youtu.be/n_8S2tCUvkM. Trailer del documentario

versi, si sono opposti al progetto di sterminio, e a rischio della propria vita hanno salvato altre vite e protetto i perseguitati». Una riflessione specifica sulle figure dei "Giusti" porta a sottolineare l'importanza in ogni momento storico della responsabilità individuale e della libertà di scelta di ciascuno, che può agire con giustizia e mostrarsi solidale con gli altri, mettendo a rischio la propria vita per salvare chi è minacciato e oppresso. Così hanno agito Gino Muratori, Giuseppina Frignani Muratori e Giudo Morganti, che hanno salvato le famiglie Finzi e Rimini [Finzi 2006], così hanno agito don Enzo Boni Baldoni, che ha nascosto la famiglia Modena", e gli abitanti di Nonantola.

Accanto al dolore e alla consapevolezza dell'ingiustizia subita, le testimonianze di Cesare Moisè Finzi e di Bruno Modena manifestano una profonda gratitudine. Nello studio della Shoah, insieme alla memoria del male commesso, acquista rilievo anche la memoria del bene compiuto, basata sulla centralità della collaborazione, della responsabilità individuale, della condivisione, della solidarietà. Per il giudice Moshe Bejski ${ }^{12}$ «i Giusti hanno dimostrato che il male assoluto non

\footnotetext{
Legge 20 luglio 2000, n. 211 Istituzione del "Giorno della Memoria" in ricordo dello sterminio e delle persecuzioni del popolo ebraico e dei deportati militari e politici italiani nei campi nazisti.
}

11 Cfr. Fai del bene buttalo ai pesci, documentario a cura di Alessandra Fontanesi e Andrea Mainardi, Istoreco 2017.

12 Salvato da Oskar Schindler, è stato il principale artefice del Giardino dei Giusti. 
vince mai completamente» e l'unico sollievo possibile davanti alla Shoah è dato «dall'opera degli uomini giusti che in ogni epoca hanno il coraggio di affrontare il male e che ogni volta salvano il mondo» [Nissim 2011, 6].

\section{Bibliografia}

Bensoussan G. 2013, Storia della Shoah, Firenze: Giuntina

Finzi C. M. 2006, Qualcuno si è salvato ma niente è più stato come prima, Cesena: Il Ponte Vecchio

Nissim G. 2017, La bontà insensata. Il segreto degli uomini giusti, Milano: Mondadori

Picciotto L. 2017, Salvarsi. Gli Ebrei d'Italia sfuggiti alla Shoah. 1943-1945, Torino: Einaudi

Ricoeur P. 2003, La memoria, la storia, l'oblio, Milano: Raffaello Cortina

Sarfatti M. 2005, La Shoah in Italia. La persecuzione degli ebrei sotto il fascismo, Torino: Einaudi

Violi P. 2014, Paesaggi della memoria, Milano: Bompiani

Voigt K. 2002, Villa Emma, Ragazzi ebrei in fuga 1940-1945, Firenze: La Nuova Italia

\section{Risorse}

Una surreale normalità. Modena e l'Italia al tempo delle leggi antiebraiche

https://unasurrealenormalita.it

Pietre d'inciampo Parma

https://pietredinciampoparma.it

Per non dimenticare il bene. Percorso della memoria trai "Giusti tra le Nazioni" in

Emilia-Romagna

www.giustiemiliaromagna.it

Fondazione centro di documentazione ebraica contemporanea www.cdec.it

Fondazione Villa Emma www.fondazionevillaemma.org

Gariwo. La foresta dei Giusti www.gariwo.net 